Algebraic $\& \mathcal{G}$ Geometric Topology

Volume 2 (2002) 381-389

Published: 22 May 2002

ATG

\title{
Exotic smooth structures on nonpositively curved symmetric spaces
}

\author{
BORIS OKUN
}

\begin{abstract}
We construct series of examples of exotic smooth structures on compact locally symmetric spaces of noncompact type. In particular, we obtain higher rank examples, which do not support Riemannian metric of nonpositive curvature. The examples are obtained by taking the connected sum with an exotic sphere. To detect the change of the smooth structure we use a tangential map from the locally symmetric space its dual compact type twin.
\end{abstract}

AMS Classification 53C35; 57T15, 55R37, 57R99

Keywords Locally symmetric space, exotic smooth structure, duality, tangential map

\section{Introduction}

The purpose of this note is to show how tangential maps between dual symmetric spaces, constructed in [11], can be used to obtain exotic smooth structures on a compact locally symmetric space of noncompact type. In particular, we construct higher rank examples, which then by Eberlein-Gromov Rigidity Theorem do not support a Riemannian metric of nonpositive curvature. This answers in the negative the question, due to Eberlein, of whether a smooth closed manifold, homotopy equivalent to a nonpositively curved one, admits a Riemannian metric of nonpositive curvature. Rank 1 examples with the same property were independently constructed in [1].

The simplest way to attempt to change the smooth structure on a manifold is to take the connected sum with an exotic sphere. The question remains, however, of whether this procedure actually changes the structure. This is where tangential maps become useful.

The paper is organized as follows. Section 2 contains general statements, showing how to produce nontrivial (up to concordance) smooth structures of the 
above sort on the domain of a tangential map from the ones on its range. In Section 3, we apply these techniques to nonpositively curved locally symmetric spaces and obtain nondiffeomorphic structures.

Though our arguments are straightforward generalizations of some of the arguments in [5], [7], and are probably well known to specialists, for reader's convenience we present them in some detail.

The material in this paper is part of the author's 1994 doctoral dissertation written at SUNY Binghamton under the direction of F. T. Farrell. I wish to thank him and S.C. Ferry for numerous useful discussions. The author was partially supported by NSF grant.

\section{$2 \quad$ Tangential maps and smooth structures}

In this section, we show that certain types of smooth structures remain nontrivial under tangential maps. The arguments here are very close to those of [7], though our statements are more general.

First, we recall the following very useful lemma:

Lemma 2.1 ([8, p. 509]) Let $\xi$ be a $k$-dimensional vector bundle over an $n$ dimensional space, $k>n$. If the Whitney sum of $\xi$ with a trivial bundle is trivial, then $\xi$ itself is trivial.

Let $\mathbb{D}^{n}$ denote the closed ball of radius 1 in the Euclidean $n$-dimensional space.

Lemma 2.2 Let $k: M^{n} \rightarrow N^{n}$ be a tangential map between two closed smooth $n$-dimensional manifolds. Then $M^{n} \times \mathbb{D}^{n+1}$ is diffeomorphic to a codimension 0 submanifold of the interior of $N^{n} \times \mathbb{D}^{n+1}$.

Proof Let $i: N^{n} \rightarrow N^{n} \times \mathbb{D}^{n+1}$ be the standard inclusion $i(x)=(x, 0)$ and $p: N^{n} \times \mathbb{D}^{n+1} \rightarrow N^{n}$ be the projection on the first factor $p(x, y)=x$.

Consider the composition $i \circ k: M^{n} \rightarrow N^{n} \times \mathbb{D}^{n+1}$. By Whitney Embedding Theorem this composition can be approximated by an embedding $w: M^{n} \rightarrow$ $N^{n} \times \mathbb{D}^{n+1}$, such that $w$ is homotopic to $i \circ k$.

Let $\nu$ denote the normal bundle of the manifold $M^{n}$ considered as a submanifold of $N^{n} \times \mathbb{D}^{n+1}$ via $w$. By definition of the normal bundle, we have:

$$
w^{*}\left(\tau\left(N^{n} \times \mathbb{D}^{n+1}\right)\right)=\nu \oplus \tau\left(M^{n}\right)
$$


On the other hand,

$$
\tau\left(N^{n} \times \mathbb{D}^{n+1}\right)=p^{*}\left(\tau\left(N^{n}\right)\right) \oplus \epsilon^{n+1}
$$

where $\epsilon^{n+1}$ denotes the $(n+1)$-dimensional trivial bundle.

Combining these two equations together, we obtain:

$$
\nu \oplus \tau\left(M^{n}\right)=w^{*}\left(p^{*}\left(\tau\left(N^{n}\right)\right)\right) \oplus \epsilon^{n+1}
$$

Since by construction $w \simeq i \circ k$, we have $w^{*}=(i \circ k)^{*}=k^{*} \circ i^{*}$. Note that $i^{*} \circ p^{*}=(p \circ i)^{*}=i d$ and $k^{*} \tau\left(N^{n}\right)=\tau\left(M^{n}\right)$ since the map $k$ is tangential. It follows that

$$
\nu \oplus \tau\left(M^{n}\right)=\tau\left(M^{n}\right) \oplus \epsilon^{n+1}
$$

i.e. the bundle $\nu$ is stably trivial.

By Lemma $2.1 \nu$ is trivial itself; therefore, the tubular neighborhood of $M^{n}$ in $N^{n} \times \mathbb{D}^{n+1}$ is diffeomorphic to $M^{n} \times \mathbb{D}^{n+1}$.

Let $Z^{n}$ be a closed, connected $n$-dimensional manifold. There is a unique homotopy class of degree 1 maps from $Z^{n}$ to $\mathbb{S}^{n}$, which we will denote by $f_{Z}$. One can obtain such a map by collapsing to a point the exterior of the small neighborhood of a point in $Z^{n}$.

It turns out that a tangential map induces a map on suspensions going in the "wrong" direction.

Lemma 2.3 (cf. [7, Corollary 3.11]) Let $k: M^{n} \rightarrow N^{n}$ be a tangential map between $n$-dimensional manifolds. Then there exist a map

$$
g: \Sigma^{n+1} N^{n} \rightarrow \Sigma^{n+1} M^{n}
$$

such that the suspension $\Sigma^{n+1} f_{N}$ and the composite $\left(\Sigma^{n+1} f_{M}\right) \circ g$ are homotopic as maps from $\Sigma^{n+1} N^{n}$ to $\Sigma^{n+1} \mathbb{S}^{n}=\mathbb{S}^{2 n+1}$.

Proof Fix base points $x_{0} \in M^{n}$ and $y_{0} \in N^{n}$. Let $B$ denote a small neighborhood of $x_{0}$ in $M^{n}$. By general position, we may assume that the embedding

$$
F: M^{n} \times \mathbb{D}^{n+1} \rightarrow N^{n} \times \mathbb{D}^{n+1}
$$

of Lemma 2.2 has the additional property that:

$$
\operatorname{Im}(F) \cap\left(y_{0} \times \mathbb{D}^{n+1}\right) \subseteq F\left(B \times \mathbb{D}^{n+1}\right)
$$

The suspensions $\Sigma^{n+1} M^{n}$ and $\Sigma^{n+1} N^{n}$ can be identified as the following quotient spaces:

$$
\Sigma^{n+1} M^{n}=M^{n} \times \mathbb{D}^{n+1} /\left(M^{n} \times \partial \mathbb{D}^{n+1} \cup B \times \mathbb{D}^{n+1}\right)
$$




$$
\Sigma^{n+1} N^{n}=M^{n} \times \mathbb{D}^{n+1} /\left(M^{n} \times \partial \mathbb{D}^{n+1} \cup y_{0} \times \mathbb{D}^{n+1}\right)
$$

Let $*$ denote the point in $\Sigma^{n+1} M^{n}$ corresponding to the subset $M^{n} \times \partial \mathbb{D}^{n+1} \cup$ $B \times \mathbb{D}^{n+1}$ in the first formula. Define $g: \Sigma^{n+1} N^{n} \rightarrow \Sigma^{n+1} M^{n}$ by

$$
g(y)= \begin{cases}F^{-1}(y), & \text { if } y \in F\left(\left(M^{n}-B\right) \times \operatorname{Int}\left(\mathbb{D}^{n+1}\right)\right) \\ *, & \text { otherwise. }\end{cases}
$$

It is easy to check that $\Sigma^{n+1} f_{N}$ and $\left(\Sigma^{n+1} f_{M}\right) \circ g$ are homotopic.

Now let us turn our attention to smooth structures.

Definition 2.4 Let $Z$ be a compact closed topological manifold, and let $Z_{1}$, $Z_{2}$ be two smooth structures on $Z$. $Z_{1}$ is said to be concordant to $Z_{2}$ if there is a smooth structure $\bar{Z}$ on $Z \times[0,1]$ such that $\partial_{-} \bar{Z}=Z_{1}$ and $\partial_{+} \bar{Z}=Z_{2}$.

Let $\Sigma^{n}$ be an exotic $n$-dimensional sphere, [8]. One of the ways to produce a (possibly) nontrivial smooth structure on a smooth $n$-dimensional manifold $Z^{n}$ is to take the connected sum $Z^{n} \# \Sigma^{n}$. The following lemma shows that, at least for this type of variation of smooth structures, tangential maps preserve nontriviality of smooth structures.

Lemma 2.5 Let $k: M^{n} \rightarrow N^{n}$ be a tangential map between n-dimensional manifolds and assume $n \geq 7$. Let $\Sigma_{1}$ and $\Sigma_{2}$ be homotopy $n$-spheres. Suppose that $M^{n} \# \Sigma_{1}^{n}$ is concordant to $M^{n} \# \Sigma_{2}^{n}$, then $N^{n} \# \Sigma_{1}^{n}$ is concordant to $N^{n} \# \Sigma_{2}^{n}$.

We note that there is no condition on the degree of the map $k$ above.

Proof Recall $[9$, p. 194] that concordance classes of smooth structures on a smooth manifold $Z^{n}(n>4)$ are in one-to-one correspondence with homotopy classes of maps from $Z^{n}$ to Top / O denoted by $\left[Z^{n}\right.$, Top / O]. The space Top / $\mathrm{O}$ is an infinite loop space (see [3, p. 215]). In particular, Top / $\mathrm{O}=$ $\Omega^{n+1} Y$ for some space $Y$. We have suspension isomorphism $\left[Z^{n}\right.$, Top $\left./ \mathrm{O}\right] \cong$ $\left[\Sigma^{n+1} Z^{n}, Y\right]$, so the smooth structures on $Z^{n}$ can be thought of as (homotopy classes of) maps from $\Sigma^{n+1} Z^{n}$ to $Y$. In this way a connected sum $Z^{n} \# \Sigma^{n}$ gives rise to a map from $\Sigma^{n+1} Z^{n}$ to $Y$, which we will denote $z_{\Sigma}$. Using the standard sphere $\mathbb{S}^{n}$ in place of $Z^{n}$ we see that an exotic sphere $\Sigma^{n}$ itself corresponds 
to a map $s_{\Sigma}: \mathbb{S}^{2 n+1} \rightarrow Y$. The naturality of this construction and Lemma 2.3 imply that the diagram

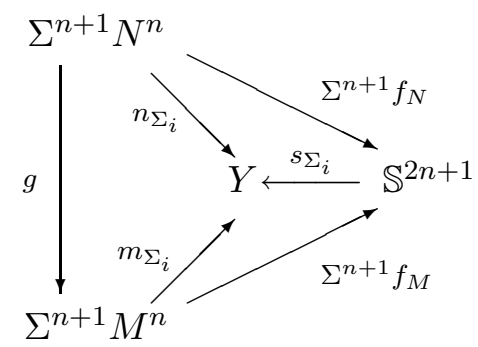

is homotopy commutative for $i=1,2$. In particular, we see that $n_{\Sigma_{i}} \simeq m_{\Sigma_{i}} \circ g$.

Since the connected sums $M^{n} \# \Sigma_{1}^{n}$ and $M^{n} \# \Sigma_{2}^{n}$ are concordant, the maps $m_{\Sigma_{1}}$ and $m_{\Sigma_{2}}$ are homotopic. It follows that the maps $n_{\Sigma_{1}}$ and $n_{\Sigma_{2}}$ are homotopic, and therefore the connected sums $N^{n} \# \Sigma_{1}^{n}$ and $N^{n} \# \Sigma_{2}^{n}$ are concordant.

\section{$3 \quad$ Exotic smoothings of locally symmetric spaces}

In this section, we apply the results of the previous section to the tangential map constructed in [11]. We show how to produce nonstandard smooth structures on the nonpositively curved symmetric spaces.

We will use notation from [11]. Let $G$ be a real semisimple algebraic linear Lie group (a subgroup of $\mathrm{GL}(n, \mathbb{R})$ ) and $K$ be its maximal compact subgroup. Let $G_{c}$ denote the complexification of $G$ and let $G_{u}$ denote a maximal compact subgroup of $G_{c}$. Let $\Gamma$ be a cocompact torsion-free discrete subgroup of $G$. The space $X=\Gamma \backslash G / K$ is a locally symmetric space of noncompact type and the space $X_{u}=G_{u} / K$ is a symmetric space of compact type. Slightly abusing terminology, we will refer to the spaces $X$ and $X_{u}$ as dual symmetric spaces.

The tangential map is provided by the following theorem:

Theorem 3.1 [11] Let $X=\Gamma \backslash G / K$ and $X_{u}=G_{u} / K$ be dual symmetric spaces. Then there exist a finite sheeted cover $X^{\prime}$ of $X$ (i.e. there is a subgroup $\Gamma^{\prime}$ of finite index in $\Gamma$ with $\left.X^{\prime}=\Gamma^{\prime} \backslash G / K\right)$ and a tangential map $k: X^{\prime} \rightarrow X_{u}$.

We will also use the following three rigidity results.

Theorem 3.2 (The Strong Mostow Rigidity Theorem [10]) Let $X_{1}$ and $X_{2}$ be compact locally symmetric spaces of noncompact type such that the universal 
cover of $X_{1}$ has no 2-dimensional metric factor projecting to a closed subset of $X_{1}$. Then any isomorphism from $\pi_{1}\left(X_{1}\right)$ to $\pi_{1}\left(X_{2}\right)$ is induced by a unique isometry (after adjusting the normalizing constants for $X_{1}$ ).

Theorem 3.3 (Eberlein-Gromov Strong Rigidity Theorem [4], [2]) Let $X$ be compact locally symmetric space of noncompact type such that all metric factors of $X$ have rank greater than 1 . Let $M$ be a closed connected nonpositively curved Riemannian manifold. Then any isomorphism from $\pi_{1}(X)$ to $\pi_{1}(M)$ is induced by a unique isometry (after adjusting the normalizing constants for $X)$.

Theorem 3.4 (Farrell-Jones Topological Rigidity Theorem [6]) Let $M$ be a closed connected $m$-dimensional Riemannian manifold with nonpositive sectional curvature, let $N$ be a topological manifold (possibly with boundary) and let $H: N \rightarrow M \times \mathbb{D}^{n}$ be a homotopy equivalence which is a homeomorphism on the boundary. Assume $m+n \neq 3,4$. Then $H$ is homotopic rel boundary to a homeomorphism from $N$ to $M \times \mathbb{D}^{n}$. i

The following lemma provides a connection between concordance and diffeomorphism classes of smooth structures for locally symmetric spaces.

Lemma 3.5 (cf. [5]) Let $X=\Gamma \backslash G / K$ be a compact orientable symmetric space of noncompact type such that the universal cover $G / K$ of $X$ has no 2 -dimensional metric factor projecting to a closed subset of $X$ and assume $\operatorname{dim} X \geq 7$. Let $\Sigma_{1}$ and $\Sigma_{2}$ be homotopy spheres of the same dimension as $X$. Suppose $X \# \Sigma_{1}$ is diffeomorphic to $X \# \Sigma_{2}$. Then $X \# \Sigma_{1}$ is concordant either to $X \# \Sigma_{2}$ or to $X \#\left(-\Sigma_{2}\right)$.

Proof The argument given to prove Addendum 2.3 in [5] holds almost literally in our setting. For completeness we give the argument here.

We can assume that the connected sums of $X$ with $\Sigma_{1}$ and $\Sigma_{2}$ are taking place on the boundary of a small metric ball $B$ in $X$, so we think of $X \# \Sigma_{1}$ and $X \# \Sigma_{2}$ as being topologically identified with $X$, and the changes in the smooth structure happen inside $B$. Let $f: X \# \Sigma_{1} \rightarrow X \# \Sigma_{2}$ be a diffeomorphism.

First we consider a special case, where $f: X \rightarrow X$ is homotopic to the identity. Thus we have a homotopy $h: X \times[0,1] \rightarrow X$ with $\left.h\right|_{X \times 1}=f$ and $\left.h\right|_{X \times 0}=i d_{X}$. Define $H: X \times[0,1] \rightarrow X \times[0,1]$ by $H(x, t)=(h(x, t), t)$. Note that $H$ is a homotopy equivalence which restricts to a homeomorphism on the boundary. Therefore, by Farrell--Jones Topological Rigidity Theorem, $H$ is homotopic rel 
boundary to a homeomorphism $H^{\prime}: X \times[0,1] \rightarrow X \times[0,1]$. We put the smooth structure $\left(X \# \Sigma_{2}\right) \times[0,1]$ on the range of $H^{\prime}$ and, by pulling it back along $H^{\prime}$, obtain the smooth structure $N$ on the domain of $H^{\prime}$. Since, by construction, $H^{\prime}: N \rightarrow\left(X \# \Sigma_{2}\right) \times[0,1]$ is a diffeomorphism, $\left.H^{\prime}\right|_{X \times 1}=f$ and $\left.H^{\prime}\right|_{X \times 0}=i d_{X}$, $N$ is a concordance between $X \# \Sigma_{1}$ and $X \# \Sigma_{2}$.

The general case reduces to the above special case as follows. If $f: X \rightarrow$ $X$ is an orientation preserving homeomorphism, then, by the Strong Mostow Rigidity Theorem, $f^{-1}$ is homotopic to an orientation preserving isometry $g$. Since one can move around small metric balls in $X$ by smooth isotopies, $g$ is homotopic to a diffeomorphism $g^{\prime}: X \rightarrow X$ such that $\left.g^{\prime}\right|_{B}=i d_{B}$. Since $X \# \Sigma_{2}$ is obtained by taking the connected sum along the boundary of $B$ and $\left.g^{\prime}\right|_{B}=i d_{B}$ and $\left.g^{\prime}\right|_{M-B}$ is a diffeomorphism, it follows that $g^{\prime}: X \# \Sigma_{2} \rightarrow X \# \Sigma_{2}$ is also a diffeomorphism. Therefore the composition $g^{\prime} \circ f: X \# \Sigma_{1} \rightarrow X \# \Sigma_{2}$ is a diffeomorphism homotopic to the identity and it follows from the previous special case that $X \# \Sigma_{1}$ and $X \# \Sigma_{2}$ are concordant.

If $f$ is an orientation reversing homeomorphism, then similar argument produces a diffeomorphism $g^{\prime}: X \# \Sigma_{2} \rightarrow X \#\left(-\Sigma_{2}\right)$ and it follows that $X \# \Sigma_{1}$ is concordant to $X \#\left(-\Sigma_{2}\right)$.

Our main result is the following theorem:

Theorem 3.6 Let $X=\Gamma \backslash G / K$ and $X_{u}=G_{u} / K$ be compact dual symmetric spaces such that the universal cover $G / K$ of $X$ has no 2-dimensional metric factor projecting to a closed subset of $X$ and assume $\operatorname{dim} X \geq 7$. Let $X^{\prime}$ be the oriented finite sheeted cover of $X$ the existence of which was established by Theorem 3.1. Let $\Sigma_{1}$ and $\Sigma_{2}$ be homotopy spheres of the same dimension as $X$.

If the connected sum $X_{u} \# \Sigma_{1}$ is not concordant to both $X_{u} \# \Sigma_{2}$ and $X_{u} \#\left(-\Sigma_{2}\right)$ then $X^{\prime} \# \Sigma_{1}$ and $X^{\prime} \# \Sigma_{2}$ are not diffeomorphic.

Proof Suppose the connected sums $X^{\prime} \# \Sigma_{1}$ and $X^{\prime} \# \Sigma_{2}$ are diffeomorphic. Then, by Lemma 3.5, $X^{\prime} \# \Sigma_{1}$ is concordant either to $X^{\prime} \# \Sigma_{2}$ or to $X^{\prime} \#\left(-\Sigma_{2}\right)$. It follows from Lemma 2.5 that either $X_{u} \# \Sigma_{1}$ is concordant to $X_{u} \# \Sigma_{2}$ or to $X_{u} \#\left(-\Sigma_{2}\right)$, which contradicts the hypothesis. This contradiction proves the theorem.

Applying this theorem to [11, Example 4], we obtain the following corollary: 
Corollary 3.7 Let $G=G_{c}$ be a complex semisimple Lie group, and let $X=$ $\Gamma \backslash G_{c} / G_{u}$ and $X_{u}=G_{u}$ be compact dual symmetric spaces such that $\operatorname{dim} X \geq$ 7. Let $X^{\prime}$ be the oriented finite sheeted cover of $X$ the existence of which was established by Theorem 3.1. Let $\Sigma_{1}$ and $\Sigma_{2}$ be two nondiffeomorphic homotopy spheres of the same dimension as $X$. Then the connected sums $X^{\prime} \# \Sigma_{1}$ and $X^{\prime} \# \Sigma_{2}$ are not diffeomorphic.

Proof Since the group $G_{c}$ is a complex Lie group, we do not have to worry about 2-dimensional metric factors; hence, according to Theorem 3.6 it suffices to show that the connected sum $G_{u} \# \Sigma_{1}$ is not concordant to both $G_{u} \# \Sigma_{2}$ and $G_{u} \#\left(-\Sigma_{2}\right)$. We recall that the tangent bundle of a Lie group is trivial. Therefore the constant map $G_{u} \rightarrow \mathbb{S}^{\operatorname{dim} G_{u}}$ is tangential, and the required statement follows from Lemma 2.5 .

The above corollary provides examples of exotic smooth structures on locally symmetric space of higher rank, therefore by Eberlein-Gromov Rigidity Theorem 3.3 these examples do not admit Riemannian metric of nonpositive curvature. One can apply Theorem 3.6 to real and complex hyperbolic manifolds to obtain exotic smoothings of Farrell-Jones [5],[7]. This is trivial in the real case, since the dual space is a sphere, and requires rather intricate analysis of the smooth structures on the complex projective space (see [7]) in the complex case. The case of the quaternionic hyperbolic manifolds seems still to be open.

\section{References}

[1] C S Aravinda, F T Farrell, Rank 1 aspherical manifolds which do not support any nonpositively curved metric, Comm. Anal. Geom. 2 (1994) 65-78

[2] Werner Ballmann, Mikhael Gromov, Viktor Schroeder, Manifolds of nonpositive curvature, Birkhäuser Boston Inc., Boston, MA (1985)

[3] J M Boardman, R M Vogt, Homotopy invariant algebraic structures on topological spaces, Lecture Notes in Math. 347, Springer-Verlag, Berlin-New York (1973)

[4] P Eberlein, Rigidity of lattices of non-positive curvature, Ergodic Theory \& Dynamical Systems 3 (1983) 47-85

[5] F T Farrell, L E Jones, Negatively curved manifolds with exotic smooth structures, J. Amer. Math. Soc. 2 (1989) 899-908

[6] F T Farrell, L E Jones, Topological rigidity for compact nonpositively curved manifolds, Proc. Sympos. Pure Math. 54 (1993) 229-274 
[7] F T Farrell, L E Jones, Complex hyperbolic manifolds and exotic smooth structures, Invent. Math. 117 (1994) 57-74

[8] M Kervaire, J W Milnor, Groups of homotopy spheres: I, Annals of Math. 77 (1963) 504-537

[9] R C Kirby, L C Siebenmann, Foundational essays on topological manifolds, smoothings and triangulations, Ann. of Math. Studies 88, Princeton University press, Princeton, NJ (1977)

[10] G D Mostow, Strong rigidity of locally symmetric spaces, volume 78 of Ann. of Math. Studies, Princeton University press, Princeton, NJ (1973)

[11] B Okun, Nonzero degree tangential maps between dual symmetric spaces, Alg. Geom. Topol. (2001) 709-718

Department of Mathematical Sciences, University of Wisconsin-Milwaukee Milwaukee, WI 53201, USA

Email: okun@uwm.edu

Received: 14 January 2002 Revised: 16 May 2002 